\title{
DEMONSTRATIONS OF THE ACTIVITY THEORY FRAMEWORK FOR RESEARCH IN INFORMATION SYSTEMS
}

\author{
Dr Kate Crawford \& Dr Helen Hasan \\ School of Economics and Information Systems, \\ University of Wollongong \\ Wollongong 2522, Australia \\ Email: hasan@uow.edu.au
}

\begin{abstract}
The holistic and insightful nature of the (cultural-historical) Activity Theory has led to its use by several researchers as a suitable vehicle for understanding and analysis in many areas of IS research and practice. This paper demonstrates the application of Activity Theory to the study of socio-technical systems which mediate complex, collective activities in the modern workplace and in everyday life. Vignettes from five ongoing research projects are reported in order to illustrate not only the explanatory power of the Activity Theory research framework but also its use in determining appropriate methods used to manage the data collection and analysis processes as well as its interpretation., The paper demonstrates the variety of IS topics where an Activity Theory based approach is able to add richness and insight.
\end{abstract}

\section{INTRODUCTION}

The field of Information Systems (IS) is concerned with both technical and human aspects of organisational and social systems. IS covers a broad range of possible topics of study and the application of a wide variety of research methods. In some areas of IS research there is a need for a holistic theoretical framework to provide a basis for research into complex socio-technical phenomena. Although the (cultural-historical) Activity Theory was proposed long before the advent of computers, its holistic and insightful nature has the potential to provide a suitable vehicle for understanding and analysis in many areas of IS research and practice. A growing band of researchers recognise that the theory provides a rich holistic understanding of how people do things together with the assistance of sophisticated tools in complex dynamic environments (Hasan 1999).

This paper explores the explanatory, analytical and interpretive power of Activity Theory for the study of socio-technical systems which mediate complex, collective activities in the modern workplace. One advantage of the theory is that it supports analysis of the dialectic interactions between people and the ways technologies shape and are shaped by human activity. The paper describes the framework and discusses the circumstances under which an Activity Theory framework could be employed for research in IS, as well as in the related fields of Human-Computer Interaction and Knowledge Management. Some of the research techniques and methods of data collection and analysis that are appropriate within an Activity Theory Framework are also presented.

Vignettes from five-ongoing research projects are reported to illustrate the variety of IS topics where an Activity Theory framework is being used. In each case, the methods used to conduct the research and manage the data collection and analysis processes are presented through the Activity Theory 
Framework. The paper concludes with a discussion of the relevance and suitability of Activity Theory as a framework for current complex problems in IS.

\section{AN OVERVIEW OF ACTIVITY THEORY}

Activity Theory is a social-psychological theory that has its roots in the work of the Russian psychologist Vygotsky during the first half of the $20^{\text {th }}$ century. Vygotsky's important insight into the dynamics of consciousness was that it is essentially subjective and shaped by the history of each individual's social and cultural experience (Vygotsky 1978). In addition, Vygotsky saw human activity as quite distinct from that of non-human entities in that it is mediated by tools, the most significant of which is language. Vygotsky's work was continued by others, amongst them Leontiev who developed a conceptual framework for a complete theory of human activity (Leontiev 1981). According to Leontiev (1981), activity is a system that has structure, its own internal transitions and transformations, and its own development.

The notion of "activity" is interpreted from the theory of Leontiev (1981) which is, in turn, based on the psychology of Vygotsky (1978). The constructs of Vygotskian psychology that are fundamental to activity theory concern those elements of activity and consciousness that distinguish humans from other beings. Essentially, Vygotsky (1978) defined human activity as a dialectic relationship between subject and object, i.e. a person working at something. In this dynamic, purposeful relationship the 'always active' subject learns and grows while the object is interpreted and reinterpreted by the subject in the ongoing conduct of the activity.

Vygotsky (1978) also proposed an 'instrumented' structure of activity within a 'system of interrelationships' between people (Verenikina \& Gould 1998). In other words all human activity is purposeful, is carried out through the use of 'tools' and is essentially social. Vygotsky also believed that tools play a mediating role in all human activities and mental processes which can only be understood in terms of the tools and signs that mediate them. Tools expand our possibility to manipulate and transform objects but also restrict what can be done within the limitation of the tool, which, in turn, often stimulates improvements to the tool (Verenikina \& Gould 1998). Wertsch (1985) believed that this rich concept of mediation was Vygotsky's most unique contribution. Wartofsky (1979) proposed that the tools that mediate human activities could be primary (tangible, external or physical), secondary (internal, semiotic or mental), or tertiary (schematics where mind and culture act together such as environments or ecosystems). This describes the full spectrum of tools and is not a rigorous classification or separation of types. With the advent of the Internet, computer-based technologies fall into all three types.

Leontiev (1981) proposed that "activity" should be placed at the top of the hierarchy shown in Figure 1 , associated sustained human endeavour with a long-term purpose and strong motives. This is a conceptual level above that at which most business analysis takes place, which is at the level of actions, undertaken towards specific, and often short-term, goals. Under certain conditions, conscious actions can be driven to a lower level of automation, often in computer systems, as they become standardised as operations. An activity is comprised of sets of actions (towards specific goals) and operations (routine and well known habitual cognitive or behavioural processes, now commonly the domain of IT systems). Whereas an activity is defined by purpose and motive, and is typically a long-term affair, actions are more planned with specific goals and a more limited time span. Actions are not meaningful in themselves unless they are part of an activity. For example, it makes no sense to drive to work (an action) unless there is a work activity to go to. 


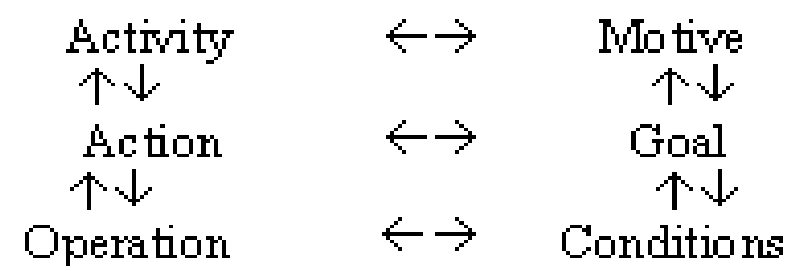

Figure 1. The definitive hierarchy of Leontiev (1981)

There may be legitimate alternative sets of actions that can enable the successful performance of an activity, for example: it is common practice in IS development to assess the feasibility of different design solutions to an organisational problem and then choose one solution to implement based on a cost benefit analysis. However there may be instances where it is feasible to allow concurrent different solutions (i.e. different sets of actions) for an activity under different circumstances (e.g. in different countries where cultures vary or in different divisions of a company). It is important however to have a common understanding of the object (purpose) of the activity at the top of the hierarchy.

Kuutti and Virkkunen's research (1995) has used activity systems as a representation of the common object of organisational work which cannot be studied by reducing the scope to one or another element, but where a minimum meaningful system as a whole should be taken as the unit of analysis and intervention. Engeström (1987) gave a more concrete expression to this structure in the triangular representation, shown in Figure 2, which is commonly used to depict an activity. The core of an activity is a dialectic relationship between subject (human) and object (purpose) mediated by tools and community. This is a two-way concept of mediation where the capability and availability of tools mediates what is able to be done and tools, in turn, evolve to hold the historical knowledge of how the communities behaves and is organised. This is particularly powerful when the tools are computer-based (Kaptelinen 1996). The formal, or informal, rules and division of labour of the community, in which the activity occurs, also dynamically mediate the subject-object relationship.

To be able to analyse complex interactions and relationships, Engeström (1987) proposes a new unit of analysis he calls a human activity system. According to Engeström the system is object-oriented, tool mediated, and culturally mediated. Engeström suggests that it is the internal tensions and contradictions of such an activity system, which includes both historical continuity and locally situated contingency that are the motive for change and development. Dynamic cycles of expansive learning are of crucial importance to the historical understanding of activity systems. These cycles, shown in Figure 2, combine the process of internalisation and externalisation where internalisation is the reproduction of culture by socialising and training individuals to be members of the activity system, and creative externalisation is the creation of new artefacts through innovations. 


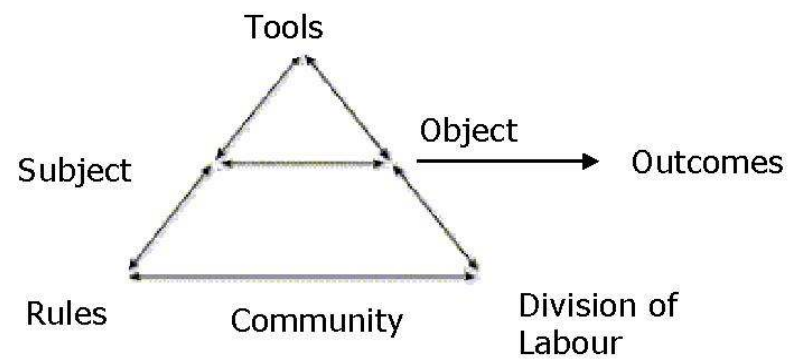

Figure 2. The subject-object relationship, which defines an activity, is mediated by tools and community through rules and division of labour. The subject may be individual or collective and outcomes of the activity are distinct from its object/purpose.

In addition to Engeström's structure of activity (Figure 2) and Leontiev's hierarchy of activity, actions and operations (Figure 1), there are several groups of researchers (Kuutti \& Virkunnen 1995; Hasan \& Gould 2001, Engeström 1999) who use frameworks of interrelated activities to represent complex organisational situations as shown in Figure 3. Engeström (ibid) uses the Cycle of Expansive Learning shown in Figure 4 to demonstrate the development that takes place within an activity system due to contradictions within and between related activities. Taken together the three aspects of human activity will be used to analysis and present the case described in the following section of the paper.

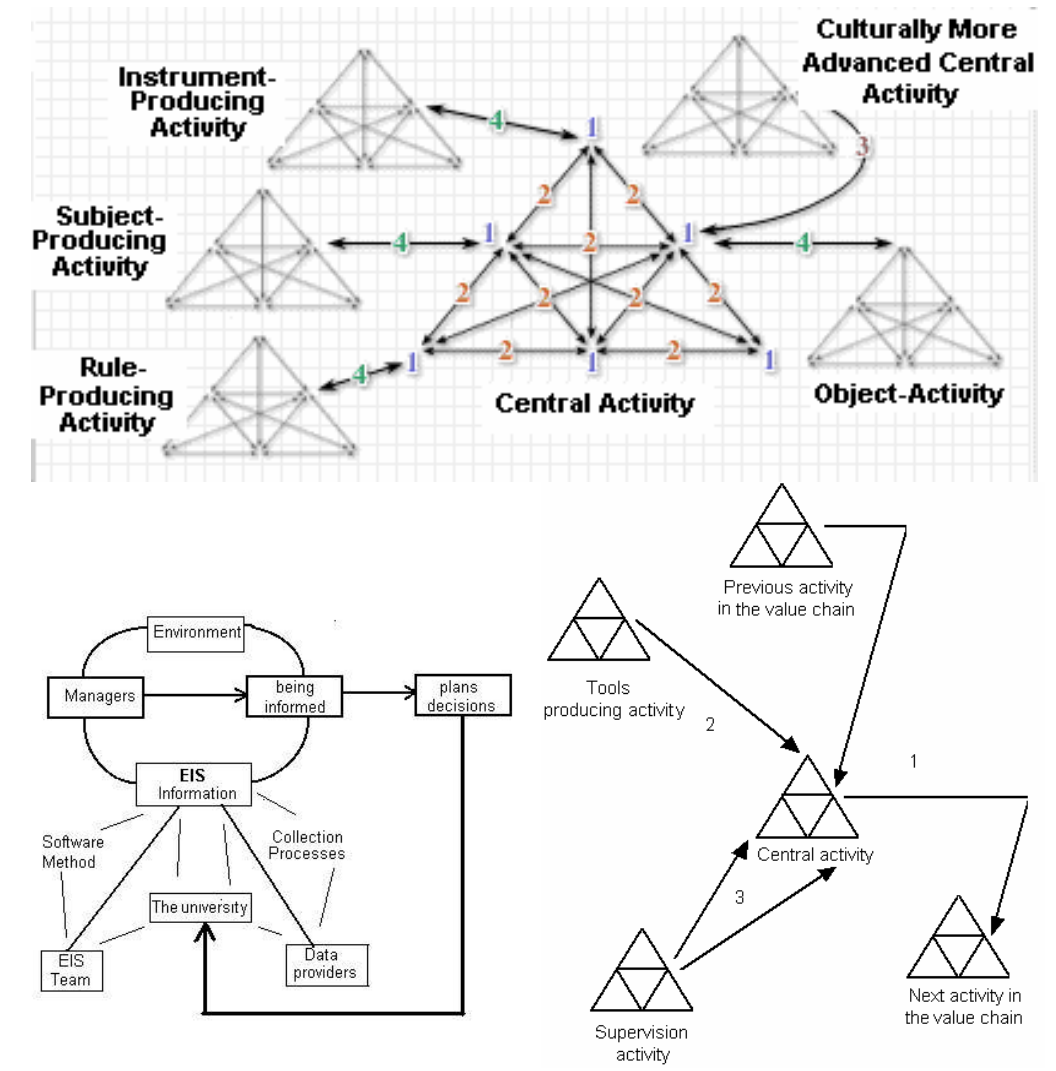

Figure 3. Sets of interrelated activities in the research of Engeström (1999), Hasan \& Gould (2001) and Kuutti \& Virkunnen (1995) 


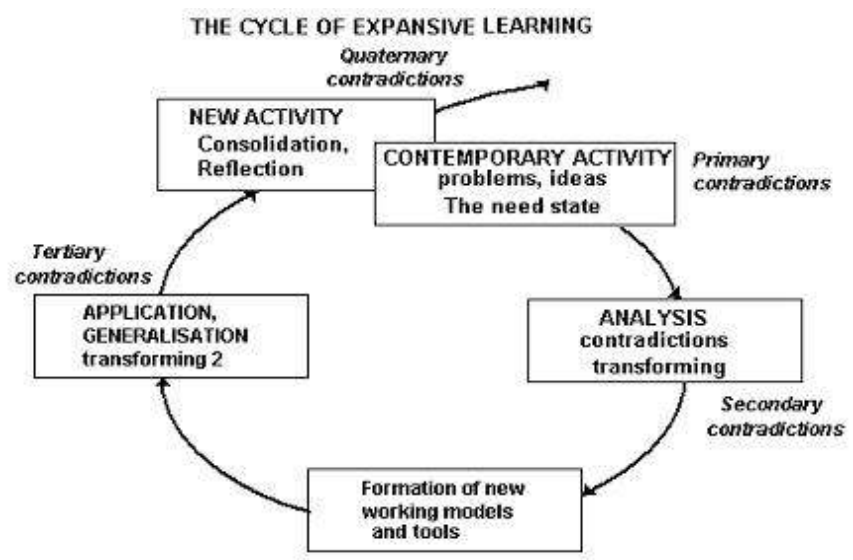

Figure 4. The Cycle of Expansive Learning (Engeström 1999)

As already stated, an activity is defined by the tool-mediated relationship between subject and object - that is, between the doer and their purpose. The mediation is a mutual development of both the activity and the tools which including primary (physical) tools, secondary tools (ideas, models etc) and tertiary tools, such as the community within which the activity takes place. The capability and availability of tools, mediates what can be done in the community at all three levels.

\section{WHY USE ACTIVITY THEORY?}

A major reason for the use of Activity Theory in IS research is that it provides a well developed framework for analysing the complex dynamics of the settings that are investigated. These settings characteristically involve interacting human and technical elements - -subjective, creative and emotionally experienced human behaviours together with automated, logical, analytic and routine modes of activity that can be embedded in modern technology. Within this framework the core of an activity is the subject-object dialectic and it is the experience of the authors that this dialectic view of socio-technical systems is appropriate in complex settings. Research in this field needs to take into consideration the dynamic interplay over time of information and communication technologies, emerging cultural trends, patterns of human experience, activity and use, and the related cultural and organizational processes that emerge, or are inhibited, as the dimensions and purpose of the whole system of work activity changes. Activity theory with its focus on accumulating factors that affect the subjective interpretations, the purpose, and sense making of individual and group actions and operations, also provides a useful paradigm for the ways in which human experience, needs and creativity shape the design and effectiveness of emerging technologies

\section{WHERE TO USE ACTIVITY THEORY}

During the 1980s and 1990s a growing body of researchers began to recognise the relevance of Activity Theory to the study of information systems (IS) and related disciplines. Some representative publications include the work of Kuutti (1991), Gould (1998), Korpela et al (2000) and Star (1996) in Information Systems as well as Bodker (1990), Bannon (1990), Kaptelinin (1992), Draper (1993) in 
the related field of Human Computer Interaction. This work stems from an awareness by the researchers of the unique position of the computer as a primary, secondary and tertiary tool following the availability of the personal computer as a support tool for individuals with software such as wordprocessing, spread sheets and other decision support systems initially and then for groups through email and other web-based applications of the Internet.

Information systems in the 1960s and 1970s had revolutionised the way organisations function by automating the day-to-day processing of billions of individual operational transactions, thereby collecting and storing huge quantities of information-producing data. There is however another class of computer-based information systems that support creative work practices rather than automate those that are routine. Following the impact of the personal computer and the Internet in the latter part of the twentieth century, the twenty-first century has seen computer-based connectivity, systems and devices become indispensable tools for almost everything we do. This has resulted in a complex and ever-changing work-life environment enabled by complex socio-technical tools involving ever more sophisticated and ubiquitous applications which are being developed to take advantage of the rapid advances in digital technologies. The complex phenomena associated with the socio-technical systems that have emerged in this environment are prime targets for research using Activity Theory as an underlying framework because Activity Theory provides a framework for analysing data about emerging patterns of human activity in terms of changing purposes, awareness, focus of attention, and tools. Rather than the view that information systems are composed of discrete interacting elements, Activity Theory sees at their core the dialectic parings of subjective and objective, learning and doing, individual and collective, social and technical as well as tacit and explicit knowledge.

While many developments in Information Systems are targeted at the global mass market or multinational corporate world, these are not the ones that most interest Activity Theory researchers. In highly commercial firms, government departments and educational institutions, desirable outcomes are commonly achieved, not at the organisational or individual level, but at the group level in work units, cross-functional teams or communities of people who have come together with a common interest. (Linger \& Warne 2001). In her ground-breaking thesis, Zuboff (1988) anticipated this trend noting the distinctive contextual characteristics of applications, such as decision support systems (DSS) and personal productivity tools, and assigned them to the category of systems which 'informate' rather than 'automate' work practices. More recently with the convergence of communication and information technologies, such systems also shape patterns of interaction and communication. The system designs are also increasingly shaped by factors associated with communication needs and social relationships.

\section{HOW TO USE ACTIVITY THEORY}

Traditionally scholastic activity has been associated with 'ivory tower' institutions and concerns that are removed from the practical issues of work activity. In searching for research methods appropriate to the study of activities involving dynamic and complex socio-technical systems, the authors are in accord with the notion of a "New Scholarship" (McNiff 2000) that is used to describe a new way of knowing that meets the everyday needs of people working in real-life situations. Real-life practices are messy, uncontrolled and unpredictable and are seriously separated from the sanitised world of abstract theorising. McNiff (ibid) proposes that learning from experience, although not highly valued by the academy, can be reinforced through intellectual study. He contrasts this kind of intellectual work to traditional forms of scholarship, which values facts and information without reference to context or human purposes and experiences. 
The approach to this research is rooted in reflection-in-action, which implies that the research will be participatory, evolutionary, contextual, holistic and developmental. The developmental research method is a disciplined investigation conducted in the context of the creation and implementation of a product or program, in this case a socio-technical system and model, for the purpose of improving either the thing being developed or developer. It is holistic, contextual and evolutionary, where a prototype model is constructed and used with the target group. The dynamics of the system are analysed through participatory observation before the prototype (both the technology and the emerging new social system) is revised.

The approach is strongly influenced by the expanding spiral of learning in the developmental work research (DWR) approach (Engeström 1987), where communities of learning and practice are viewed as activity systems (Virkkunen \& Kuutti 2000). DWR provides a dynamic framework that can accommodate a multifaceted analysis of the community members, their motives and purpose for belonging, their relationships within the community and the tools that mediate community activity. In this research the tools include technology together with social and learning processes. Discipline is imposed on the investigation by the analysis of each case as an activity system, in the tradition of Activity Theory. In an activity system, the unit of analysis is the work activity itself seen as a dialectic of the subject (person or persons doing the work) and the object and purpose of the doing. Work activity is culturally, technically and historically located and, where IS is involved, there is usually a complex mix of interrelated activities in one activity system. The following is a Activity Theory framework for representing and analysing such situations

\section{AN ACTIVITY THEORY FRAMEWORK FOR IS RESEARCH}

This framework takes the concept of activity is used as the unit of analysis to explain what individuals or small groups of people do in a variety of contexts when supported by socio-technical systems. In all research there are conceptually two activity systems: that of the researchers and that which forms the object of their research. From a DWR perspective these activity systems are so closely related that, at the core of the research activity, is a dynamic subject-object dialectic where the researchers may actively participate or intervene in the activities which are the object of their research. In the IS tradition of action research this lack of detachment of the researchers often poses a dilemma (McNiff 2000). However in this framework it is a basic assumption that provides a balance of depth and breadth to the scope of a study to include a richness of understanding (depth) and the inclusion of multiple interrelated and dynamic factors (breadth) that is not possible in other approaches.

Scaffolding onto this basis the framework then emphasises work/learning activity system being studied is generally comprised of the following components:

1. the purpose to which members of the work community direct their activity

2. individual workers/learners, their colleagues and co-workers/learners

3. the conceptual models, tools and equipment they use, and

4. the rules, culture and context that govern how they work, and learn through their work

The Activity Theory framework also recognises the unique and pervasive nature of the computer as a primary, secondary and tertiary tool underpinning an enormous variety of organisational information systems. This is reflected in the ways in which researchers in IS use computers. For example computers are used as primary tools to search for information, as secondary tools to create reports and analyse data, and increasingly web based virtual interactive spaces are used as tertiary tools to support and structure quality interaction between people in a working IS system. 
Each vignette will now be presented within this framework under the following headings:

1. The object of the research activity - i.e. the work/learning activity system being studied in terms of the subject-object dialectics of the constituent activities and how these activities make up an activity system.

2. The subjects/people of the activities - their composition and growth as individuals and groups as the activities evolve and change

3. The purpose of those activities - the various, sometimes conflicting, motives that may be involved

4. The primary, secondary and tertiary tools used for the work/learning activities being studied

5. The culture and context within which they occur

6. The tools of the research activity

7. Research interventions and its evolution.

\section{ILLUSTRATIONS OF ACTIVITY THEORY IN IS RESEARCH}

The research described in the vignettes below illustrates several aspects of activity associated with research and development of information systems. In some the system is the tool that supports the research process in others the system is the object of research and also a tool for collecting data of various kinds. Thus, in vignette 1 the research activity has a focus outside a technical system on structuring data collection using the Q-Sort method and analysing the resulting information through statistical analysis. In Vignette 2 the focus is more the way new tools are used to assist in creating and analysing models of complex activities. Vignettes 3 and 4 describe research that analysis the complex activity of developing information systems of various kinds and the final vignette (5) discusses the emergent research into patterns of human collaborative activity within one of the systems.

\section{Vignette 1: A Q-study to Elucidate User Requirements}

1. The object of the research activity - i.e. the activity system being studied

People have always sought information to assist with activities in which they are engaged. In some cases information seeking becomes an activity in its own right. Nowadays the Internet provides easy access to copious amounts of information through various general-purpose search engines. This research investigates the user requirements for search engines when they become tools for some wider activity, such as online shopping. The core activity of interest to this research is the process of information seeking on the Internet. Also of interest, are the activities for which the information sought will be used. This research uses results of a Qanalysis $^{6}$ to define archetypical activities of information seekers in the context of the Internet. (Meloche and Cheng 2005)

2. The subjects/people of the activities being studied

Almost everyone is a potential user of Internet search-engines, however most of those in the study are younger people who have grown up in the Information Age.

3. The purpose of those activities and the motives of the subjects

\footnotetext{
${ }^{6} \mathrm{http}: / /$ www.qmethod.org
} 
This research identifies the difference in purpose of different sets of information seekers and hence sees these as different types of activities.

4, The primary, secondary and tertiary tools used

The tools of interest here are Internet search engines which are primary tools for the information seeker. The q-methodology uses a statistical analysis to interpret the resulting QSort data. This is a secondary research tool as is uses a model of gathering original statements and allowing participants to subjectively sort these before a factor analysis is undertaken of the sorts.

5. The culture and context within which they occur

Among the young people used in this study the culture of using the Internet is second nature and the prime way they seek information. However, the study explores the differences between people of different groups in how they interpreted the object of the activity.

6. The tools of the research activity

The research uses the Q-methodology to elucidate an authentic set of requirements aligned to the user activities. The Q-method involves, in the first instance, a collection of statements that represent a broad range of possible views on the topic, in this case attributes of search engines. A number of typical search engine users then individually sort these statements ranking them from those they agree with most to least. These sorts are processed to identify sets of users who have similar ranking patterns. The highly ranked statements of each user set are then recommended as design requirements for search engine incorporated into the relevant activity.

7. Research interventions and its evolution

The Q-method elicits opinions as statements from participants in the research and then brings all statements back to allow participants to judge them in the sort.

Vignette 2: Organisational Knowledge Management - A Multi-level Systems Modelling Approach

1. The object of the research activity - ie the activity system being studied

The current push for managers in leveraging their organisation knowledge for competitive advantage was stimulated by the work of Nonaka (1994). His SECI model, depicted in Figure 5 , describes knowledge creation in organisations spiralling from an individual to organisational level through four processes which relate to two types of knowledge, tacit and explicit. One legacy of the popularity of this model has been the stagnation of its concepts, ie that knowledge has two distinct forms, tacit knowledge held by individuals and an explicit form stored in technology as organisational memory. Many managers see the ideal knowledge management system in their organisation as one that captures all individual tacit knowledge and makes it explicit and available to all. 


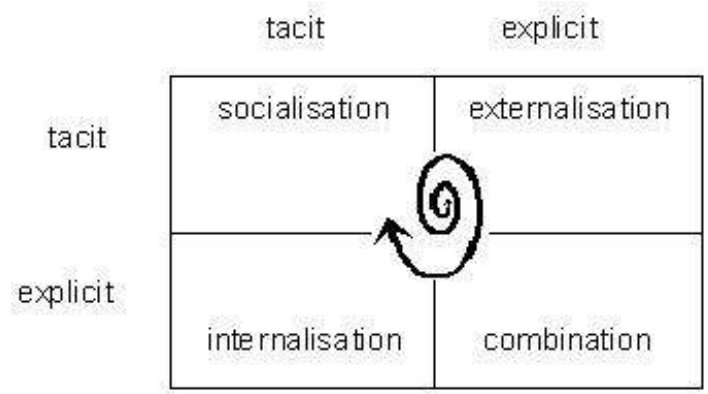

Figure 5 The SECI model of Knowledge Creation (Nonaka 1994)

The research described in this vignette addresses issues of knowledge and knowledge work in organisations taking a middle-out perspective, which is neither organisational nor individual, with the objective of opening a new discourse on knowledge work in the context of modern complex organisations (Linger et al 2005). Within the Activity Theory framework, the tacitexplicit forms of knowledge can be seen as dialectic where knowledge work always involves a synthesis of the two forms. Knowledge creation within an organisation is not seen as a linear process that starts with the individual and works towards the organisations but rather as dynamically emerging from the level of informal workgroups within an organisation's knowledge eco-system (AS5037-2005). The central activity of the activity system being studied is therefore the work unit where knowledge work is a dialectic of learning and doing. This relates on one hand to the activities of individual workers and on the other to the activities of managers who are concerned with strategic objectives, sustainability and competitive advantage.

This research has identified a severe deficiency in the way knowledge management is understood in organisations, where the focus is either on individual knowledge or on formal processes at the organisational level. Neither of these recognises that most knowledge is created through work in groups and networks that are not visible in the formal organisational structures and processes. The object of the research is to understand the complexity of how work is actually done, what is being done, who does what and how learning occurs in those activities.

2. The subjects/people of the activities being studied

This research focuses on the work group as a collective subject of knowledge work, the core of the Activity System being studied. The research is particularly interested in how self-directed groups can survive and grow in typical bureaucratic organisations and be the source of innovation enabling large organisations to be more agile and adaptable in rapidly changing environments. Individual workers and managers are subjects of secondary activities that interact with the core activity.

3. The purpose of those activities and the motives of the subjects

A thriving work group works to further the purpose of an organisation through innovation and initiative. Its main motive is often job satisfaction and self-fulfilment in contrast to the motives of individual workers and managers which may tend to be more self-centred; workers to get promoted, managers to maintain control. 
4. The primary, secondary and tertiary tools used

This research is particularly interested in the use of processes and technologies to further the purpose of particular activities. It contrasts the use of systems that are used to maintain the hierarchical command structures with those of self-directed work-groups which often make innovative use of socio-technical systems such as communities of practice, virtual work-spaces etc.

5. The culture and context within which they occur

As mentioned previously the concept of a knowledge eco-system is used to describe the culture and context of knowledge management in organisations where a balance of elements and enables is desirable but often fraught with conflict and disharmony. This follows the lead of the Australia Knowledge Management Standard (AS5037-2005).

6. The tools of the research activity

The first phase of this research used the content analysis tool, Leximancer ${ }^{7}$, to compare the use of Activity Theory with similar work done in a Task-Based approach to knowledge management (see Linger et al 2005). The current phase is using the systems dynamics modelling tool, Stella ${ }^{8}$, to simulate the interaction between individual, group, organisational and environmental knowledge activities as depicted in Figure 6. Both tools would be considered primary tools from an Activity Theory standpoint.

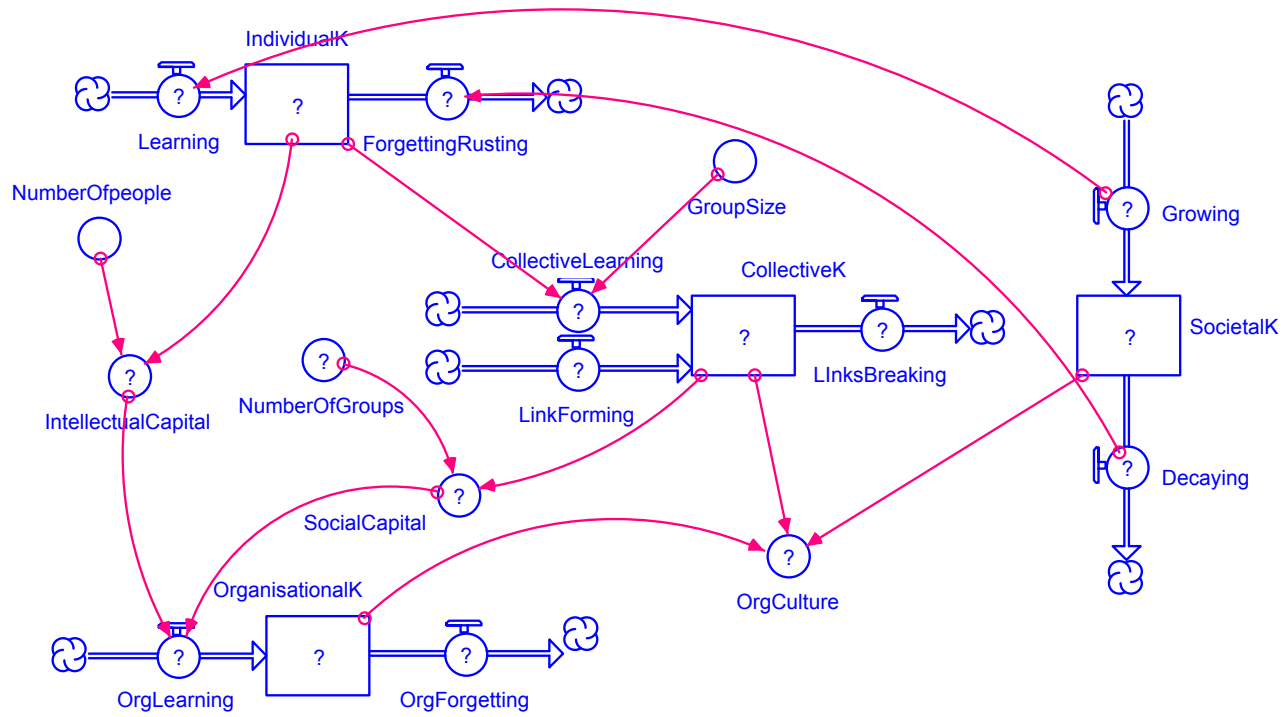

Figure 6. A Stella Model showing the structure and relationships of four levels of knowledge: individual, collective, organisational and societal.

\footnotetext{
${ }^{7}$ http://www.leximancer.com
}

${ }^{8} \mathrm{http}: / /$ www.iseesystems.com 
7. Research interventions and its evolution

The researchers will use each version of the model to get feedback from workers and managers in organisations in an evolutionary process to develop the model grounded in practice.

\section{Vignette3: Systems Development for Knowledge Creation_ The Eviva System ${ }^{9}$}

1. The object of the research activity - ie the activity system being studied

IS in a relatively new field of study that has borrowed and adapted research methodologies from older disciplines. Recent claims have been made that some original form of Design Science could be a suitable research methodology that is suited to IS as its own. In this study the activity under investigation is the evolutionary development of innovative technological systems to support web-based community groups and enable research into the behaviour and effectiveness of such communities. This process may take place over an extended period of time as prototypes are created, then used and evaluated with a community over many months giving feedback for improvements to the next use of the prototype until a stable system results. The findings of this project (Hasan 2003) have shown that evolutionary system design is a knowledge creation activity at various levels including the research process itself. Activity Theory has been used by others (e.g. Bodker 1990) to study the interaction between the activities of IT developers and the activities of the users of their products (activity outcomes). This research takes the Activity Theory analysis to another level where this interaction is seen as a tertiary tool in the knowledge creation processes of research.

2, The subjects/people of the activities being studied

The team of developers of the systems studied have a mixture of skills from technical expertise to social scientists who collect and analyse the data. The composition of the team changes over time, according the cycle of research and development, but there are usually one or two who initiate and drive the project.

\footnotetext{
${ }^{9} \mathrm{http}: / /$ www.eviva.com.au 


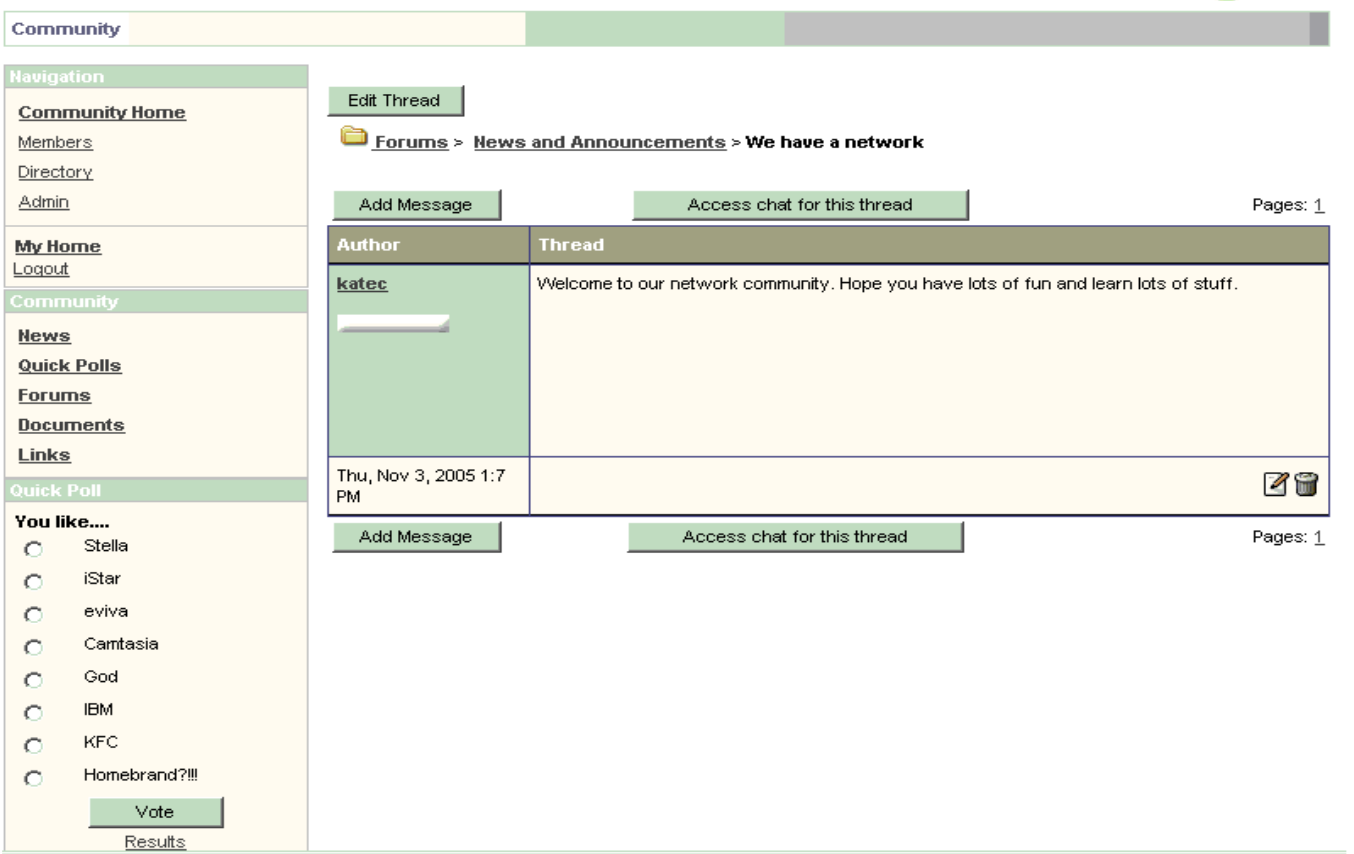

Figure 7. A screen from a networked community in Eviva showing a

Discussion Forum and community poll.

3. The purpose of those activities and the motives of the subjects

These systems are being developed both to support quality interaction and team work in online communities (see Figure 7) and to enable data collection for research in the area.

4. The primary, secondary and tertiary tools used

The system development was sponsored by a software development company and trials of the early versions of the system were undertaken as a part of the infra-structure for an existing community network. As it is rare that such systems attract substantial funding, the development takes advantage of whatever technical and research resources are available.

5. The culture and context within which they occur

The development culture is one of a small enthusiastic team with mixture of genders, ages and ethnicities. The communities using the systems are often composed of volunteers active in their community and most very grateful for the support.

6. The tools of the research activity

The research methodology is a mix of design science and action research but takes cognisance of the unique properties of computers as a tool to support and inform emergent knowledge processes. This follows the steps of design science as described by March and Smith. (1995). The development activity was iterative, user centred and responsive to user feed back in early trials. The unique environment could be considered a tertiary tool mediating the development activity. 
7. Research interventions and its evolution

This project uses the development of a knowledge support system to be used in practice but also to research complex areas of online community sustainability and growth. This metaresearch process results from interpretation and analysis of the knowledge created through the evolutionary development of innovative computer-based tools.

\section{Vignette 4: Network-Centric Organisations-simulated with the Go*Team Game}

1. The object of the research activity - i.e. the activity system being studied

Many organisations are adopting network-centric configurations in which workers leverage information and make operational decisions through the collaborative efforts of small agile and self directed teams. Go*Team is a computerised client-server team version of the ancient Chinese strategy game of Go that has been designed to simulate contexts requiring complex collaborative strategic activity by teams. Traditionally, Go is played with black and white stones on a $9 \times 9$ to $19 \times 19$ grid where individual players take turns to place their stones, Unlike standard Go, teams playing Go*Team no longer have to take turns; a team's next turn can be taken after a "relaxation time", specified via the server, regardless of whether or not the opposing team has done anything in the interim. There is also no preset command structure built into the Go*Team game. As far as the game software is concerned all team members are peers; with no predetermined roles and there is no "team leader" with more power or capabilities than other team members. The client screens for each player show only a partial view of the board so that there is a need for team members to communication their view of the board to others as well as to discuss strategies. Players on the same team make use of modern communication tools such as email, voice over IP, chat rooms and the like, to effect the cooperation and coordination they need to successfully play the game.

The specific set network-centric elements to be investigated with Go*Team include: emergent leadership, communication quality, cooperative behaviour, situation awareness, information sharing, group dynamics, trust and effective decision-making. Possible independent variables are stress and tempo (timing variable in Go*Team), uncertainty of information, the degree of training, team structure and organisation and communication mode. The activities studied in this research are sessions where teams play Go with various values of the independent variables.

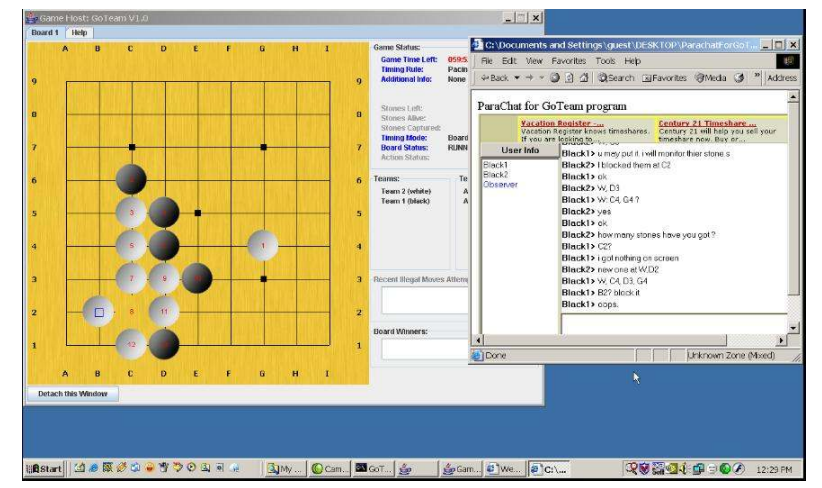

Figure 8 A still of the server screen captured showing the whole board and the Chat of the Black team during a game with students. The audio communication of the white team is also on the video recording. 
2. The subjects/people of the activities being studied

Members of highly competitive organisations such as the military and big business are typical of the people who will be recorded playing Go*Team. Of interest are the collective subjects (the teams) and the individuals within teams.

3. The purpose of those activities and the motives of the subjects

There are three possible reasons to play Go*Team. On is for the purpose of research where sets of volunteers will be used. Other objectives are to use Go*Team for training small group cooperative behaviours and another would be to profile members of an organisations for their cooperative nature.

4. The primary, secondary and tertiary tools used

The Go*Team and associated protocols of use are the tools used in the activity. Go*Team embeds players in a unique environment which balances the need to cooperate within that of competition. It can therefore be considered a tertiary tool.

5. The culture and context within which they occur

A mentioned most Go*Team will be undertaken in competitive cultures but a useful comparison will be among behaviours where team members come from diverse backgrounds.

6. The tools of the research activity

The researchers are recording Go*Team sessions using Camtasia, screen capture application. The Zing groupware meeting system ${ }^{10}$ will be used in post game debriefing sessions to collect qualitative data from participants. These are primary tools.

7. Research interventions and its evolution

As there are so many possible independent of variables that can be used, many of which are difficult to measure, experience of each Go*Team session is used to design the setting and parameters of subsequent sessions.

\section{Vignette 5: Content Analysis of Online Group Interaction}

1. The object of the research activity - i.e. the activity system being studied

This study investigates the activities of groups using an innovative web-based groupware package to support their ongoing collaborations and project development. The groups are in fact using a combination of face-to-face and online environments in carrying out these activities. However, the virtual arena for interaction extends the opportunities for shared experiences and provides an explicit and auditable record of shared interaction, patterns of emerging activity, and data about practical outcomes.

2. The subjects/people of the activities being studied

Users of the on-line community seeking to use the system as a context for quality interaction and intentional outcomes for projects within the group

3. The purpose of those activities and the motives of the subjects

The purpose of the activity for the subjects was to work in teams on joint projects or to coordinate such activities. An emerging issue in research on network -centric systems of this

${ }^{10} \mathrm{http}: / /$ www.anyzing.com/ 
kind is the need to explicitly negotiate and specify different ways of working together (rules, division of labour and community expectations) among subjects with a long cultural history of competitive and individualistic approaches to work in hierarchically organized systems. The system has been designed to provide feedback to the community as a means to support the new work practices.

4. The primary, secondary and tertiary tools used

Is this research the system (see Figure 7) is used by both researchers and community participants as a primary tool for communication and exchange, a secondary tool as it is modified and used to provide feedback and ideas and the experience shared activity embedded within the system means it functions also as a tertiary tool.

5. The culture and context within which they occur

The system is consistent with a new set of activities within the emerging digital culture. These reflect earlier experiences using older technologies but the convergence of information and communication technologies within the civil culture of developed countries has the potential to radically transform human activities and the resulting awareness and capabilities of people with a history of such experiences.

6. The tools of the research activity

The researchers collect data from the online tool where transcripts of the group interaction can be captured over time. The collected text is subjected to sophisticated content analysis tool (Leximancer) to dynamically map concepts contained in the texts as the group matures over time. The system also has the capability to map and report group activity, to poll user opinions and responses, and to facilitate user-based initiatives to structure their emerging activities and meet emerging needs. Researching quality and purposeful interaction within the system opens up a whole new field of research in IS where studies of the kind described in Vignette 1 can now be carried out within the system itself.

7. Research interventions and its evolution

In this research the activities of the groups vary greatly and the tool used supports the variety of communicative and collaborative actions in a ubiquitous way that enables the subjects to retain focus on the object of their activities. In that way the data collection and analysis can be done in a non-intrusive manner so that the researchers obtain a more genuine interpretation of the object of their study.

\section{DISCUSSION}

From the work of the authors (Hasan \& Crawford 2003a,b) and others (eg Korpella et al 2000) there is copious evidence that Activity Theory is appropriate for IS research. This paper illustrates situations where Activity Theory is eminently suitable as the underlying research framework. These situations invariably concern complex and dynamic knowledge-intensive work in groups or communities supported by socio-technical systems. In the paper, vignettes, based on real research projects, have been used to illustrate such situations as well as describing the research methods appropriately used for each study.

In each of the vignettes described above, there is an interaction of several activities, which form innovative socio-technical systems, involving groups of workers and learners acting as a supportive and interactive community environment and enabled by new information and communications 
technologies. The key element of differentiation between each of the individual or group activities is their object or motive. The purpose of the worker is markedly different from that of the researcher. However, even within a particular group a range of multifaceted objects play out, often in unexpected ways. Activity Theory provides a rich, holistic understanding of how people do things together with the assistance of sophisticate tools in complex dynamic environments where socially-constructed, collective knowledge is the predominant source of learning, creativity and innovation. An Activity Theory analysis begins with a description of the elements of these activities and the relationships between them. The seven point framework used to analyse the vignettes highlights some of these key elements.

In the technical area, the ICT systems are tools that shape activities in different ways. This is partly because the objects (purposes) of users are diverse both within and between groups and within each work activity. In addition each person in a socio-technical system brings a unique cultural history, personality and knowledge base and thus interprets, makes sense of and responds to the system individually. The diversity and complexity of human responses increases both the complexity and potential of the system. Within each activity, tensions arise between the sub elements of the system. For example, often the community expectations, rules and roles are not aligned with the potential permitted by new tools. As a result, they are used badly or not used at all in the activity system. In our view these tensions are the necessary precursors to evolution of work activity systems.

Key concepts of Activity Theory are consciousness shaped by experience and the subjectivity of human awareness. Thus, tools are what people think they are and this can vary depending on prior experiences, culture and/or purpose. As well as the interpretations of the people changing, in the research described above, the technical systems also change their function according to the object of the activity - the same system may function as primary, secondary or tertiary tool according to the activity undertaken. In complex systems such as that described in the last vignette the technology often functions in all three ways.

The expansive cycle, described by Engeström incorporates the concepts of internalisation and externalisation in a collective sense and hence is related to the creation of collective knowledge. In Activity Theory, internalisation is manifest in the reproduction of culture by socialising and training individuals to be members of the activity system. Creative externalisation is seen in innovations with the design of new artefacts and transformations of structure and process (Engeström 1999).

As already stated, an activity is defined by the tool-mediated, dialectic relationship between subject and object i.e. between the doer and their purpose. The mediation is a mutual development of both the activity and the tools which including primary (physical) tools, secondary tools (ideas, models etc) and tertiary tools, such as the community within which the activity takes place. The capability and availability of tools, mediates what can be done in the community at all three levels.

\section{CONCLUSION}

Activity Systems generally now involve complex inter-related factors that shape and are shaped by both people and technology. These situations are central to current research in IS. Activity theory provides a comprehensive, holistic and dynamic analytical framework that, in our opinion, is ideally suited as a secondary tool for research.

In this paper we have developed a seven point Activity Theory framework and used it to present and analyse the five research activities depicted as vignettes. This has demonstrated not only a method of 
applying of Activity Theory to the study of socio-technical systems which mediate complex, collective activities but also the class of IS topics where an Activity Theory based approach is able to add richness and insight. Activity Theory is relevant not only where situations have a significant historical and cultural context but also in dynamic situations where people, their purposes (objects) and their tools are in a process of rapid and constant change. Such is the case of the current environment of IS in research and practice.

\section{REFERENCES}

AS5037-2005 (2005) Australian Standard Knowledge Management, Standards Australia

Bannon L. 1990. A Pilgrims progress: From Cognitive science to Cooperative Design, AI and Society 4 259-275.

Bødker S. 1990 Through the Interface: a Human Activity Approach to User Interface Design, Hillsdale, NJ, Erlbaum.

Draper S. 1993. Activity Theory; the New Direction for HCI, International Journal of Man-Machine Studies, 36/6, pp812-21.

Ellison M. and McGrath G. M. (2001) Business Process Modelling Using Activity Theory: An Approach to Data Capture and Analysis, in H. Hasan, E. Gould, P. Larkin and L. Vrazalic eds, Information Systems and Activity Theory: Volume 2, Wollongong University Press. 143172 .

Engeström Y. (1987). Learning by expanding: An activity-theoretical approach to developmental research. Helsinki: Orienta-Konsultit.

Engeström Y. (1999) Innovative Learning in Work Teams: Analysing Cycles of Knowledge Creation in Practice, in Engeström Y. Miettinen R. Punamaki R. eds Perspectives on Activity Theory, Cambridge University Press, Cambridge UK.

Hasan H. (1999) Integrating IS and HCI using Activity Theory as a Philosophical and Theoretical Basis, Australian Journal of Information Systems 6/2 44-55

Hasan H. (2003) Knowledge Creation through Systems Development, Proceedings of ISD2003 Melbourne.

Hasan H. and Crawford K. (2003) A multifaceted approach to distributed communities of learning and practice. Proceeding of the IS Perspectives and Challenges in the Context of Globalization Conference, Athens, June 2003 for IFIP WG 8.2 and WG 9.4.

Hasan H. and Gould E. (2001) Support for the Sense Making Activity of Managers, DSS Journal, special issue on Knowledge Management, 31/3 71-86

Kaptelinin V. (1996) Activity Theory: Implications for Human-Computer Interaction in B.Nardi Ed Context and Consciousness: MIT Press, 103-116.

Korpela, M., Soriyan, H. A. and Olufokunbi, K. C. (2000): Activity analysis as a method for information systems development: General introduction and experiments from Nigeria and Finland, Scandinavian Journal of Information Systems, vol. 12, 2000, pp. 191-210.

Kuutti, K. (1991): Activity Theory and its applications to information systems research and development', in H-E. Nissen, H. K. Klein and R. Hirscheim (eds.): Information Systems Research: Contemporary Approaches and Emergent Traditions, Elsevier, Amsterdam, 1991, pp. 529-549.

Kuutti K.and Molin-Juustila T.1998 Integrating work activities into a network organisation by a document integration system in H. Hasan, E. Gould and P. Hyland Information Systems and Activity Theory: Tools in Context, Wollongong University Press.

Kuutti K and Vikkunen J (1995) Organisational Memory and Learning Network Organisation: the Case of Finnish Labour protection Inspectors, Proceedings of HICSS28.

Leontiev A.N. (1981) Problems of the Development of Mind Moscow: Progress 
Linger, H, Burstein F. and Hasan H (2005) Articulating Knowledge Work: The contributions of Activity Theory and Task-Based Knowledge Management, in G. Whymark and H. Hasan (eds,) Activity as the Focus of Information Systems Research, Knowledge Creation Press, UCQ, 71-90

Linger H. and Warne L. (2001) Making the Invisible Visible: Modelling Social Learning in Knowledge Management Context., Australasian Journal of Information Systems Special Edition, 56-66.

March, S.T. and Smith, G. (1995) Design and Natural Science Research on Information Technology, Decision Support Systems 15/4,. 251-266.

McNiff J (2000) Action Research in Organisations, Routledge, London

Meloche J and Cheng Li (2005) A Contextual approach to design and development of Interfaces for Search Engines, using Activity Theory, as the basis for informing design decisions in G. Whymark and H. Hasan (eds,) Activity as the Focus of Information Systems Research, Knowledge Creation Press, UCQ, 93-116.

Nonaka I. (1994) A Dynamic Theory of Organisational Knowledge Creation, Organisation Science $5 / 1,14-37$.

Star S.L. 1996. Working Together: Symbolic interactionism, activity theory and information systems in Y Engeström and D. Middleton (eds) Cognition and Communication at Work. Cambridge Universty Press, NY. P296-318.

Virkkunen J. and Kuutti K. (2000) "Understanding organisational learning by focusing on 'activity systems"”, Accounting, Management and Information Technology 10 291-319.

Vygotsky, L.S. (1978) Mind and Society,: Harvard University Press, Cambridge, MA

Verenikina I. and E. Gould (1998) Cultural-Historical Psychology and Activity Theory in Hasan H, Gould E. and Hyland P. (eds) Information Systems and Activity Theory: Tools in Context Wollongong University Press. 1-18.

Wertsch, J.V. (1985) The Concept of Internalization in Vygotsky's Account of the Genesis of Higher Mental Functions in J.V. Wertsch (ed) Culture, Communication and Cognition: Vygotskian Perspectives, Cambridge University Press.

Warne L. Ali I., Hasan H. (2003) Social Learning through Activity Theory, in H. Hasan I. Verenikina and E Gould. eds Information Systems and Activity Theory Volume 3 Expanding the Horizon, UOW Press, pp 96-120.

Wartofsky, M.W. (1979): Perception, Representation, and the Forms of Action: Towards an Historical Epistemology, (1973) Models, Representation and the Scientific Understanding, Boston: D. Reidel Publishing Co.

Zuboff S (1988) In the Age of the Smart Machine, Basic Books. 
